\title{
Acceleration and Particle Field Interactions of Cosmic Rays: Formalism
}

\author{
${ }^{1}$ A. Tawfik, ${ }^{1}$ A. Saleh, ${ }^{2}$ M.T. Ghoneim and ${ }^{3}$ A.A. Hady \\ ${ }^{1}$ Egyptian Center for Theoretical Physics (ECTP), MTI University, Al-Mukattam, Cairo, Egypt \\ ${ }^{2}$ Department of Physics,Faculty of Science, Cairo University, Cairo, Egypt \\ ${ }^{3}$ Department of Astronomy, Space Science and Meteorology, Faculty of Science, Cairo University, Cairo, Egypt
}

\begin{abstract}
The origin and acceleration theories of Ultrah High Energy Cosmic Rays (UHECRs) are still mesterious problem in modern physics and astrophysics. The acceleration of ultra high energy cosmic rays is conjectured to occur through interactions with electromagnetic fields in different astrophysical objects, like magnetic matter clumps, besides the well-known shock waves and elastic Fermi mechanisms. Based on this model, a considerable portion of the dynamics, that derives a charged particle parallel to a magnetic field B and under the influence of a force $\mathrm{F}$, is assumed to be composed of acceleration by a non-magnetic force $\mathrm{F}_{\|}$ and a gyromotion along $\mathrm{B}$ direction plus drifts in the direction of $\mathrm{F}_{\perp}$. The model and its formalism are introduced, section 2 and section 3. Various examples for drift motions and accelerating forces are suggested, section 4. The configuration of the cosmic electromagnetic in different astophysical plasma aspects could play intrinsic role in the acceleration process of UHECRs. In a forthcoming work, a quantitative estimation of the energy gained by charged cosmic rays in various astrophysical objects will be evaluated.
\end{abstract}

Keywords: Ultra High Energy Cosmic Rays (UHECR), Greisen-Zatsepiti-Kuzmin (GZK), Cosmic Microwave Background (CMB), Active Galactic Nuclei (AGN), Gamma-Ray Bursts (GRB)

\section{INTRODUCTION}

One of the greatest mysteries in modern physics is the origin of Ultra High Energy Cosmic Rays (UHECR). The current acceleration technologies are unable to come up with such energy scales. In order to compare, let us mention the several observatories in which cosmic rays with energies $>10^{20} \mathrm{eV}$ have been registered (Abraham et al., 2007; Yoshida and Dai, 1998; Nagano and Watson, 2000; Takeda et al., 1998; Teshima, 2001). From the theoretical point of view, the protons, which are dominant components of UHECRs, would posses energy values much higher than the so-called Greisen-ZatsepitiKuzmin (GZK) cutoff $1019.7 \mathrm{eV}$. They are conjectured to suddenly lose their energies in photopion production processes with the Cosmic Microwave Background (CMB) radiation (Greisen, 1966; Zatsepin and Kuzmin, 1966; Nagano and Watson, 2000). This model is known as the top-down scenario.
In this study, we study the acceleration of cosmic charged particles starting from low energies, i.e., bottomup scenario. The kinematics of such particles that move parallel to a magnetic field B is assumed to be composed of an acceleration through a non-magnetic force $F_{\|}$and a gyromotion along the direction of $\mathrm{B}$ field plus the drift (s) in the direction of $\mathrm{B} \times \mathrm{F}_{\perp}$, as illustrated in Fig. 1. The drift motions are not depending on the Larmor radius and therefore can exist even in cold plasmas. This would partly explain why a non-relativistic formalism is explicitly given here. The drift motions are the summation of all types of the perpendicular velocities stemming from different non-magnetic forces. On the other hand, the stochastic acceleration according to the second-order Fermi mechanism apparently stems from the accumulation of a small velocity change with nonnegligible random velocity components in each elastic collision with the magnetic cloud.

Corresponding Author: Tawfik, A., Department of Egyptian Center for Theoretical Physics (ECTP), MTI University, Cairo-Egypt Physics, Research Center for Einstein Physics, Freie-University Berlin, Berlin, Germany 


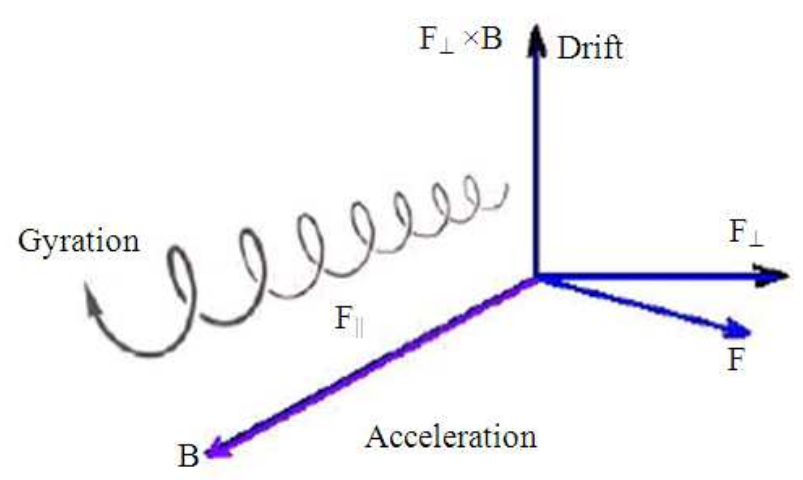

Fig. 1. Trajectory of motion of a charged particle in a nonuniform magnetic field under the influence of a nonmagnetic force $\mathrm{F}$

The magnetic cloud can be considered as a clump of a homogeneously distributed matter moving with Alfven wave velocity $\mathrm{v}_{\mathrm{A}}=\mathrm{B}_{0} \sqrt{4 \pi \rho}$, where $\mathrm{B}_{0}$ and $\rho$ are strength of background magnetic field and background matter density, respectively. With background we mean the cloud's local frame of reference. The final acceleration occurs as a consequence of the field interactions and multiple scatterings of the charged particles with the electromagnetic irregularities.

To answer the question about the possibility of finding both magnetic and electric fields in various astrophysical objects, let us first recall that the magnetic fields are basic characters of the cosmic matter clumps. The existence of electric fields would be explained by the cloud's plasma properties (Parkes, 2004). The Active Galactic Nuclei (AGN) and Gamma-Ray Bursts (GRB) are often considered as plausible sources for the astrophysical accelerators of extragalactic UHECR (Murase and Beacom, 2010). The highly magnetized neutron stars (Ghisellini et al., 2008) and the structure formation shocks (Inoue, 2008) are conjectured as possible sources. Also, the spinning black holes have been suggested (Prescod-Weinstein et al., 2009) to accelerate the cosmic charged particle. The existence of lectric fields in all these astrophysical objects depends on various parameters. On one hand, they would sanctify that the cosmic rays likely participate in various field interactions. On the other hand, they might be baptized as Eevatrons. They would provide energy scales up to several hundred $\mathrm{EeV}\left(10^{21} \mathrm{eV}\right)$. In this discussion, it is not argued that the electric fields should necessarily be long-ranged.

In literature, it is widely accepted that the large-scale steady astrophysical electric fields have not been confirmed, yet. In present work, we do not touch this point, explicitly. The model is not exclusively depending on the existence of large-scale electric fields in astrophysical context. In a forthcoming work (Tawfik et al., 2010; Tawfik and Saleh, 2010), we closely deal with this subject. For the time being, it is essential to mention that a general force $\mathrm{F}$ is assumed here. It can appear as magnetic, electric, gravitational forces. Also it is clear that the diffusion of cosmic rays in large-scale electric fields is also a subject of astrophysics (Klein, 2003). As discussed above, unipolar inductor sources like pulsars, planet magnetospheres and the electric fields in quasiperpendicular shock waves can provide plausible candidates for electric fields. On the other hand, the large electrical conductivity of space plasmas prevents to sustain stationary and steady large-scale electric fields. The electric fields in space plasmas can only appear as (i) transient phenomena as in magnetic reconnection situations or (ii) fluctuating fields as in plasma turbulence in magnetized gases, where $\mathrm{E} \sim 0+\delta \mathrm{E}$, with a vanishing ensemble average $\langle\delta \mathrm{E}\rangle$. As a consequence, four basic types of particle acceleration processes in space plasmas are relevant: (I) acceleration by magnetic reconnection. (II) Stochastic acceleration by resonant particles interactions with the plasma waves. This is a modern version of second order Fermi acceleration. (III) Diffusive shock acceleration. This is the modern version of first order Fermi acceleration. (VI) Conversion of relativistic bulk motion to relativistic particles.

Hillas (1984) introduced a widely celebrated approach. Magnetic field interactions are assumed to accelerate the cosmic charged particles. In present work, we suggest an extension to this pioneering work. Both electric and stochastic Fermi interactions are also taken into consideration. Furthermore, we assume a general configuration for the electromagnetic fields. We are not exclusively considering the very special configuration that magnetic and electric forces are perpendicular to each other. The latter apparently limits the gyration to a concrete minimum value. It is conjectured that various interaction types might be possible in one astrophysical object. The final energy is then accumulated over successive acceleration steps.

In present work, both first and second-order Fermi mechanisms remain playing their essential roles. It is worthwhile to mention that the energy gained according to Fermi mechanisms explicitly depends on the relative particle's velocity $\mathrm{v}$ with respect to the velocity of the rest frame $u$ of the magnetic clouds and/or matter clumps. Various types of field interactions have been added to the stochastic Fermi interactions. This reflects among others the quantum properties of the cosmic particles. An illustration of this approach is given in Fig. 2. 


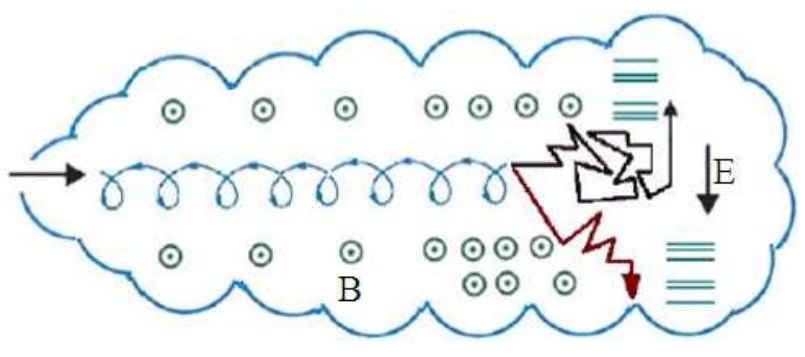

Fig. 2. An illustration of the model applied in this letter with ideal configuration. The magnetic cloud embeds regions of static and spatiotemporal-varying electromagnetic fields, so that the charged particles likely experience both field interactions and elastic scattering

In general, it turns to be no longer a trivial task to suggest a new mechanism in order to explain the acceleration of cosmic charged particles to such ultra high energy. Many constrains have to be taken into account. One of these constrains would be the requirement that the particle must be imprisoned inside the range of the active field while it is being accelerated. Therefore, one has to be aware with the particle gyroradius. Other constrains would include the strengths of accelerating fields and the time interval till the particle reaches EeV-energy-scale. Also, one has to take into consideration the various fluctuations and the possibilities for energy loss. In present work, we highlight the properties of cosmic particles (The most Dominant Components of Ultra High Energy Cosmic Rays are the Protons, (Kotera and Olinto, 2011)). They are electrically charged and eventually carry other quantum numbers, like mass and angular momentum. Over their long path to the Earth, they go through various plasma fields and elastic/shock wave collisions. Therefore, various types of electromagnetic field interactions have to be added to Fermi acceleration mechanisms in order to compute the final energy.

\subsection{Model}

In present work, we suggest a model, which fulfills many of the constrains discussed in the introduction. Since electric and magnetic fields can also exist in cold plasmas, we explicitly introduce a non-relativistic formalism. The translation into a relativistic version is straightforward (Schlickeiser and Jenko, 2010). We therefore leave it to an upcoming work, which will be devoted to the quantitative calculation of the energy gained by cosmic charged particles.

Let us consider a charged particle with mass $m$ and static electric charge $q$ in an electromagnetic field characterized be $\mathrm{E}$ and $\mathrm{B}$. $\mathrm{E}$ and $\mathrm{B}$ being globally conserved electric and magnetic fields, respectively. This assumption does not prevent that both fields can be varying, at least locally. We also assume that particle undergoes the well-known elastic (Fermi and Yang, 1949) and shock (Blandford and Eichler, 1987) Fermi mechanisms. In addition to the elasttic (collisionless) scattering, which mainly changes the particle's initial velocity $v$ by $\pm 2 \mathrm{u}$, we assume that the particle simultaneously interacts with the magnetic and nonmagnetic forces, Fig. 1. These forces are not necessarily uniform or constant.

First, we like to refer to the fact that magnetic field in electromagnetic interactions is not necessarily participating in the acceleration mechanism. Accordingly, it merely rotates or redirects the particle's motion. This is apparently restricted to the so-called " $\mathrm{E} \times$ B" drift velocity in uniform electromagnetic fields. In this case, the electric and magnetic forces are simply given by $\mathrm{qE}$ and $\mathrm{qv} \times \mathrm{B}$, respectively. The guiding center is given as $v E=(E \times B) / B^{2}$. The subscript $E$ in this case refers to the fact that $\mathrm{E}$ derives a drift velocity independent on the background properties but referring to the interactions with $\mathrm{B}$. The uniform configuration of the electromagnetic fields would limit the discussion to very special astrophysical phenomenon. But the galactic dipolar fields and loops, for instance, are poorly described by this special kind of the configurations.

In present work, we study general configurations of electromagnetic fields. They are not necessarily perpendicular to each other. Furthermore, we take into consideration that they are spatially and temporally varying. The effects of gravitational fields should not be entirely excluded, especially, when the gravitational forces of extremely gigantic astrophysical objects are dominant (Prescod-Weinstein et al., 2009).

\subsection{Formalism}

\subsubsection{Cosmic Charged Particle Resting in the Magnetic Cloud}

We first assume that the cosmic charged particle in the cloud's frame of reference fulfills two conditions; resting at a certain point, where the electromagnetic potential is nearly vanishing and entering the cloud with an initial velocity. These two cases are graphically illustrated in Fig. 2. It is conjectured that the forces affecting the particle are based on Lorentz (E, B) and gravitational $\mathrm{G}$ fields. In natural units, the equation of motion of a charged particle reads: 
$m \frac{d v}{d t}=q[E(r, t)+v+B(r, t)]+m G(r, t)$

where, $\mathrm{G}$ is the effective gravitational acceleration. For simplicity, we assume that $r$ gives a certain one-dimensional direction that is parallel to the lines of B-field. According to the assumption, which is frequently applied in literature, that the gravitational fields are much weaker than the electromagnetic ones, especially for light particles, then the particle moves with the velocity:

$v=\sqrt{-\frac{2 q \phi}{m}}$

And acceleration Equation 4:

$\mathrm{a}=\frac{1}{\sqrt{-2 \mathrm{qm} \phi}}\left(\phi \mathrm{I}+\mathrm{q} \frac{\mathrm{d} \phi}{\mathrm{dt}}\right)=\sqrt{\frac{2 \mathrm{qE}}{\mathrm{mr}}} \mathrm{v}$

where, $\varphi$ and I being the electric potential and current along r, respectively. Obviously, the electromagnetic fields-in these expressions-are conjectured to be static and uniform. In Equation 3 and seeking for simplicity, we assume that $\mathrm{E}$ makes an angle $\theta=\pi / 4$ with $\mathrm{B}$. Furthermore, we assume that both $\mathrm{v}$ and $\mathrm{B}$ are parallel, i.e., in Magneto Hydrodynamics (MHD) $\mathrm{E}_{\perp}=\mathrm{J} / \sigma-\mathrm{V} \times$ $\mathrm{B}$, where $\mathrm{V}$ is the velocity of the background plasma. $\mathrm{J}$ is the current density and $\sigma$ is the charge conductivity. Then, the cosmic charged particle would gain a nett acceleration resulting from the parallel acceleration and drifts across B-field. Its direction is given by the parity image of $\mathrm{E}$ on a mirror allocated along the lines of Bfield. The same is valid for the particle's guiding center:

$a=\sqrt{\frac{\sqrt{2}}{m r}\left(E_{\|}-v \times B\right) v}$

where, an ideal MHD has been assumed. $\mathrm{R}$ is the distance covered by the particle, which can be related to the Larmor radius $r_{L}$ (Paul, 2006) $r_{L}$ is defined as the radius of the cyclotron motion derived by the magnetic force. The motion of the changed particles under the influence of these static electromagnetic fields can be decomposed into a Larmor motion in the absence of electric fields and a drift of gyration center of its rotation. This would mean that the total velocity is composed of three components $\mathrm{v}_{\perp}, \mathrm{v}_{\|}$and $\mathrm{v}_{\mathrm{D}}$. Obviously, the maximum acceleration can be obtained when $\mathrm{E}$ and $\mathrm{B}$ are parallel and the charged particle-as usual-moves along the lines of B-field.
The energy that the cosmic charged particle would gain through this field interaction reads:

$\in=\sqrt{\sqrt{2} \mathrm{mrq}\left(\mathrm{E}_{\|}-\mathrm{V} \times \mathrm{B}\right) \mathrm{v}}$

In light of Equation 5 the final motion is a nett result out from two forces. The first one is the parallel component of the electric field strength, $\mathrm{E}_{\|}$. The second force is the drift from the perpendicular component where, Fermi acceleration mechanism has been excluded.

A general expression for the drift motion would inhere in it modifications of the inhomogeneity (nonuniformity) of the electric field E:

$\mathrm{v}_{\mathrm{E}}=\left(1+\frac{\mathrm{r}_{\mathrm{L}}^{2} \nabla^{2}}{4}\right) \frac{\mathrm{E} \times \mathrm{B}}{\mathrm{B}^{2}}$

where, $E=E_{\|} \cos (\theta)+E_{\perp} \sin (\theta)$ can be replaced by arbitrary constant force. The physics remains unchanged. For example, the gravitational drift velocity $\mathrm{v}_{\mathrm{ag}}=$ $\left(\mathrm{m} / \mathrm{qB}^{2}\right) \mathrm{a}_{\mathrm{g}} \times \mathrm{B}$, where $\mathrm{a}_{\mathrm{g}}$ is the gravitational acceleration in the magnetic cloud. The dependence on mass $m$ and charge $\mathrm{q}$ implies that this drift can be neglected for light particles and its direction changes with the sign of the electric charge q. The gravitational force is proportional to the product of masses of the two interacting counterparts. Therefore, it is likely dominant, when one of the counterparts would be-for instance-a black hole. The mass of black holes would range from $\mathrm{m}_{\mathrm{BH}} \approx(0.5-1.2) \times 108 \mathrm{M}_{\square}$ (Marconi, 2006), where $M_{\square}$ is the solar mass.

We notice that Equation 6 seems to reflect a special case, in which the electric field $\mathrm{E}$ is spatially nonuniform and oriented perpendicular to the plane of $\mathrm{z}$ and $\mathrm{y}$. As given in Fig. 2, $\mathrm{z}$ is the direction of B-field. This equation would mean that the average value of drift motion of a charged particle that apparently spends more time in regions with weak $\mathrm{E}$ field strength is less than $\mathrm{E} \times \mathrm{B}$ value. The latter is to be computed at the guiding center (the center of gyration of the particle of interest) (Boris, 2013).

Within one Larmor orbit, the electromagnetic fields are assumed to be uniform. In other words, the characterizing length over which the fields vary is much smaller than rL. In this case, the gyration orbit can be approximated to a closed circle. The differential drift would result in a net current density, $I=\sum_{r} n_{r} q_{r} v_{E, r}$.

It is obvious that the field interactions are positioned within the boundaries of the magnetic cloud and therefore $r_{L} \lesssim r$ (Hillas, 1984). Then:

$r_{L}=\frac{m}{q B} v=\frac{1}{ \pm \Omega t} r$ 
where, $\Omega$ is the angular velocity. \pm stands for right- and left-handed rotation of the Larmor motion. These signs are assumed to be given by the electric charge $q . v_{\perp}$ is the velocity component which is oriented perpendicular to the $x-y$ plane. The relation between $v_{\perp}$ and the scalar $\mathrm{v}_{\mathrm{L}}$ is given by $\mathrm{v}_{\mathrm{L}}=\sqrt{\mathrm{v}_{\perp} \cdot \mathrm{v}_{\perp}}$. At an arbitrary angle $\mathrm{t}$, expression (7) would read:

$r_{L}=\frac{v}{\Omega}(\hat{x} \sin \Omega t+\hat{y} \cos \Omega t)$

It is obvious that, Equation 8 reveals a general discription to the charge particle Larmor radius.

Alternatively, we can substitute Equation 2 into Equation 3, then the energy that the charged particle would gain through the electric field $\mathrm{E}$ is given as:

$$
\in=2 q r . E
$$

Equation 9 related the energy to the electric field.

In deriving above expressions, we assume that the electric field strength $\mathrm{E}$ is responsible for the releasing of a charged particle from rest and then accelerating it to the energy given in Equation 9. Equation 9 represents the basic of the well-celebrated Hillas (1984) graph. It is worthwhile to note that this is a special case. This has been discussed above. The general case would require further treatments.

\subsection{Cosmic Charged Particle Entering the Magnetic Cloud}

When the charged particle is assumed to enter the magnetic cloud - or in general regions of magnetic irregularities -, then the initial conditions can be very much different. At a spacial distance $r_{i}$, the initial kinetic energy would take the form $\mathrm{mv}_{\mathrm{i}}^{2} / 2$. Then, Equation 1 leads to the following equation of motion:

$v_{f}^{2}-v_{i}^{2}=\frac{2}{m} q \int_{r i}^{r f} E \cdot d r$

Equation 10 apparently depends on E, exclusively i.e., the velocity difference is related to E. Since, both electric and magnetic field strengths are conjectured to be conserved, then $\mathrm{E}=-\nabla \varphi$, where $\varphi=-\mathrm{E} \mathrm{r}$ and:

$$
v_{f}^{2}-v_{i}^{2}=-\frac{2}{m} q\left[\phi\left(r_{f}\right)-\phi\left(r_{i}\right)\right]
$$

In Equation 11, we use $\mathrm{d} \varphi / \mathrm{dr}=\nabla \varphi \mathrm{dr}$. Therefore, the total energy that the charged particle gains through such an electric interaction reads:

$$
\in=2 q\left[r f \cdot E_{f}-\left(r_{i} \cdot E_{i}\right) \frac{v_{i}}{v_{f}}\right]+m r_{i} \cdot \dot{v}_{i}\left(\frac{v i}{v_{f}}\right)
$$

According to Equation 12, let us suppose that $E_{i}=E_{f}$ $=0$, i.e., no field acceleration would take place, then $\mathrm{v}_{\mathrm{i}}=$ $\mathrm{v}_{\mathrm{f}}$ and the total energy is simply given by the initial value $m \dot{v}_{i} r_{i}$. The subscripts $i$ and $f$ stand for initial and final, respectively.

To assure that the total acceleration does not dependent on the gyroradius, Equation 1 is expanded around $r_{L}=0$ and then temporarily averaged over the whole gyration period of time:

$$
\frac{\mathrm{d}}{\mathrm{dt}} \mathrm{v}_{\|}=\frac{\mathrm{q}}{\mathrm{m}} \mathrm{E}_{\|}-\frac{\mu}{\mathrm{m}} \frac{\mathrm{d}}{\mathrm{dr}} \mathrm{B}+\mathrm{v}_{\mathrm{L}} \frac{\mathrm{d}}{\mathrm{dt}} \hat{\mathrm{e}}_{\mathrm{L}}
$$

Where, $\hat{\mathrm{e}}_{\mathrm{L}}$ is the unit vector along $\mathrm{v}_{\mathrm{L}}$. Under certain assumptions, last term in both Equation 1 and 13 can be removed. In order to discuss the physical meaning of each term in Equation 13, we use-hereafter-reduced guiding center equations. Then, $\mu=\left(\mathrm{mv}_{\perp}^{2} / 2\right) / \mathrm{B} \equiv \mathrm{W}_{\perp} / \mathrm{B}$ is valid. The latter is known as"first adiabatic" invariance. It assures that the ratio of kinetic energy $\mathrm{W}_{\perp}$ of the Larmor motion (gyromotion) to the gyrofrequenvy is conserved. $\mu$ is proportional to the current due to Larmor motion through the relation $\mu=\mathrm{IA}=\left(\mathrm{q} \Omega_{\mathrm{c}} / 2 \pi\right)\left(\pi \mathrm{r}_{\mathrm{L}}^{2}\right)$. Therefore, $\mu$ gives the magnetic moment.

Assuming that the magnetic field is static and the charged particle moves on a frame, in which the perpendicular velocity forms a cyclotron (helix or spiral) motion or Larmor motion, then dotting Lorentz equation, Equation 1, by $\overrightarrow{\mathrm{v}}$ and omitting the second term lead to:

$\frac{\mathrm{d}}{\mathrm{dt}} \mathrm{m} \mathrm{v}_{\mathrm{L}}^{2}=2 \mathrm{qv}_{\perp} \cdot \mathrm{E}_{\perp}+\mathrm{mv}_{\mathrm{L}}^{2} \frac{\mathrm{v}_{\|}}{\mathrm{B}} \frac{\partial \mathrm{B}}{\partial \mathrm{r}}$

Equation 14 embraces the perpinduclar electric field $E \perp$, this reflects the fact that the electric field is only able to accelerate the cosmic charge particle not the magnetic one, where, $v_{\perp}$ is the vertical Larmor orbital velocity. Such a velocity is orthogonal to the $\mathrm{B}$-field, then the multiplying the following equation of motion Equation 15 (Paul, 2006): 
$\mathrm{m} \frac{\mathrm{dv}_{\|}}{\mathrm{dt}}=\mathrm{qE}_{\|}-\frac{1}{2} \mathrm{mv}_{\mathrm{L}}^{2} \frac{1}{\mathrm{~B}} \frac{\partial \mathrm{B}}{\partial \mathrm{r}}=-\mathrm{q} \frac{\partial \phi}{\partial \mathrm{r}}-\mu \frac{\partial \mathrm{B}}{\partial \mathrm{r}}$

By $v_{\|}$results in conservation of the total energy of the gyration center:

$$
\frac{\mathrm{d}}{\mathrm{dt}}\left[\frac{1}{2} \mathrm{mv}_{\|}^{2}+\mathrm{q} \phi+\mu \mathrm{B}\right]=0
$$

Suppose that the charged particle experiences an increasing magnetic field. As discussed above, $\mu$ is conserved. Then, $\mathrm{W}$ must be an increasing quantity. It results in an increasing $\mathrm{v}_{\perp}$-components, as well. Since B cannot add any additional work, then $\mathrm{V}_{\|}$must increase in order to conserve the total kinetic energy. The particle is trapped inside this field, i.e., a magnetic mirror, as long as $\mathrm{V}_{\|}$is sufficiently low. Otherwise, it experiences a force in the direction away from $\mathrm{B}$. Once again, the formalism is given in the non-relativistic limit. This would not prevent or exclude the relativistic one.

\subsection{Fermi Mechanisms Plus Field Interactions}

We assume that the cosmic charged particles speed through astrophysical surroundings or conditions which are offering them acceleration via electromagnetic fields. Afterward, the particles are assumed to enter through regions of magnetic irregularities. According to the second-order Fermi mechanism, the magnetic irregularities result is elastic scattering. First, we take into consideration cosmic charged particles which are accelerated from rest, i.e., their velocities increase from 0 to $\mathrm{v}$, Equation 2 . In this case, the ansatz $\mathrm{v} \rightarrow \mathrm{v} \pm 2 \mathrm{u}$ can be applied in the second-order Fermi mechanism, where both $\mathrm{v}$ and $\mathrm{u}$ are obviously aligned. Then:

$$
\epsilon_{\mathrm{F}}=\sqrt{\sqrt{2} \mathrm{mrq}\left(\mathrm{E}_{\|}-\mathrm{V} \times \mathrm{B}\right)}(\mathrm{v} \pm 2 \mathrm{u})
$$

where, $\mathrm{u}$ is the velocity of frame of reference of the magnetic cloud. Furthermore, under the assumption that the cosmic charged particle diffuses upstream or downstream (first-order Fermi mechanism) while preserving its energy in rest frame, the particle's initial energy will also be preserved proportional to $\beta=\mathrm{v} / \mathrm{u}$ ratio, i.e., $v$ in Equation 17 is to be replaced by $\beta \mathrm{u}$.

Then, we take into consideration the other case that the cosmic charged particle enters the magnetic irregularities and/or interacts with the electromagnetic fields, where its initial velocity $v_{i}$ is finite:

$$
\epsilon_{\mathrm{F}}=\frac{\mathrm{v}_{\mathrm{i}}}{\mathrm{v}_{\mathrm{f}} \pm 2 \mathrm{u}}\left[\mathrm{mr}_{\mathrm{i}} \cdot \dot{\mathrm{v}}_{\mathrm{i}}-2 \mathrm{qr}_{\mathrm{i}} \cdot \mathrm{E}_{\mathrm{i}}\right]+2 \mathrm{qr}_{\mathrm{i}} \cdot \mathrm{E}_{\mathrm{f}}
$$

In the previous expressions, for example Equation 9 and 12 , we explicitly take into consideration cases in which the acceleration occurs due to the $\mathrm{E}$ component which is oriented parallel to $\mathrm{B}$, where both electromagnetic fields are uniform. It is essential to mention here that such a uniform electric field would not be impossible to be maintained in magnetic clouds or matter clumps. The existence of uniform magnetic fields is well-known. We could assume that the conductivity would not likely be high so that the cosmic charged particles would not disturb the cloud's overall charge neutrality. To be convinced about the existence of $\mathrm{E}_{\|}$, we mention just one example. In the Earth's ionosphere (Kelley, 2009), $\mathrm{E}_{\|}$ has been registered, where the auroral particle acceleration is believed to be resulted in by this field.

According to present model, the final energy that the cosmic charged particle would gain, is accumulated from different sources. Fermi mechanisms are obvious sources. In present work, plasma field interactions are suggested as additional sources. The latter dates back to eighties of last century, when Hillas introduced his wellknown graph (Hillas, 1984) and a very special configuration that magnetic and electric forces are perpendicular to each other has been studied. This apparently would limit the gyration to a concrete minimum value. In present work, a general configuration for both electromagnetic fields is taken into consideration. Also, it is conjectured that various interaction types might be possible in one astrophysical object. We introduce examples on drift forces depending on the nonuniformality and nonhomogeneity of both electromagnetic fields. Practically, such drifts would be substituted in Equation 17 and 18.

\subsection{Examples of Electromagnetic Drifts}

Equation 6 gives a general expression for the drift velocity. To calculate other drift velocities of the gyration center due to the components of $\mathrm{E}$ whose direction is perpendicular to $\mathrm{B}$, Equation 16 can be utilized. The latter apparently assumes that $\mathrm{E}$ (or eventually any constant F) and B, as given in Equation 1, can be spatiotemporal dependent. In following subsections, we elaborate various spatiotemporal dependencies. The resulting drift forces are to be inserted into Equation 17, in order to calculate the final energy, that the cosmic charged particles would gain through Fermi and field accelerations.

\subsection{Spatially Varying Magnetic Field Strength B}

For a weak spatially varying magnetic field $B$, we assume that $\mathrm{B}$ has components in $\mathrm{z}$-direction. For simplicity, we assume that $\mathrm{B}$ varies in $\mathrm{y}$-direction, only. 
Then, the gradient with respect to $y$ implies that $B_{z}>>r_{L}$ $\mathrm{dB}_{\mathrm{z}} / \mathrm{d} \mathrm{y}$, which means that the field strength would likely have a Taylor expansion around $\mathrm{y}=0$ over region or distance $\mathrm{y} \leq \mathrm{rL}$. This leads to $\mathrm{B}_{\mathrm{z}}(\mathrm{y})=\mathrm{B}_{0}+\mathrm{y} \mathrm{dB} / \mathrm{dy}+\cdots$. Under the influence of this large and long-ranged magnetic field, the curvature of the particle's circular orbit becomes tighter so that it can be transferred into cycloid. The negative gradient is directed from stronger to weaker magnetic field regions, i.e., Larmor radius increases. According to this motion, the drift force is perpendicular to $B$ and $\nabla|B|$. Since we assume that the electric field vanishes, then $\mathrm{dv} / \mathrm{dt}=(\mathrm{q} / \mathrm{m}) \mathrm{v} \times \mathrm{B}, \mathrm{x}-$ component averaged over the gyromotion vanishes as well and averaged x-component equals Equation 19:

$\left\langle\frac{\mathrm{dv}_{\mathrm{y}}}{\mathrm{dt}}\right\rangle= \pm \mathrm{q} \frac{\mathrm{v}_{\perp} \mathrm{r}_{\mathrm{L}}}{2 \mathrm{~m}} \frac{\mathrm{dB}_{\mathrm{z}}}{\mathrm{dy}}$

As given above, we assume that the force $\mathrm{F}$ makes a $\pi / 4$ angle with the acceleration. Then $\left\langle\cos ^{2}(\mathrm{ct})\right\rangle=1 / 2$ while $<\cos \left(\Omega_{\mathrm{c}} \mathrm{t}\right)>=0$. Therefore, the"grad B" drift velocity in three dimensions (Jackson's result) reads:

$\mathrm{v}_{\Delta \mathrm{B}}= \pm \frac{\Omega \mathrm{r}_{\mathrm{L}}^{2}}{2}\left(-\frac{\nabla|\mathrm{B}| \times \mathrm{B}}{\mathrm{B}^{2}}\right)$

Equation 20 can be seen as a result from the dipole force in B gradient. It is associated with a change in the magnetic field strength $\mathrm{B}$. The direction is perpendicular to both $\nabla|\mathrm{B}|$ and B. The Ampere's law for constant or vanishing $\mathrm{E}$ reads $\nabla \times \mathrm{B}=\mu_{0} \mathrm{~J}$. Apparently, this law seems to describe the current that generates the magnetic field. Finally, we get the "grad B" drift force:

$F_{\mathrm{VB}}= \pm \mathrm{q} \frac{\mathrm{v}_{\perp} \mathrm{r}_{\mathrm{L}}}{2} \frac{\mathrm{dB}_{\mathrm{z}}}{\mathrm{dy}}$

\subsection{Non-Uniform $B$ and Vanishing $E$}

There is one further drift associated with nonuniform B and vanishing E fields. The charged particle moving along a curved magnetic field line is conjectured to experience a centripetal acceleration. To study this problem, two-dimensional cylindrical coordinate system $(\mathrm{R}, \Phi)$ is defined. The origin of the cylindrical coordinate system is located at the field line center of curvature and lying on the plane of the field lines. The radial location of the chosen point in this system is identical with the radius of local curvature of the field lines $\mathrm{R}$. Then $\hat{\Phi}=\hat{\mathrm{B}}$ and $\hat{\mathrm{B}} \cdot \nabla \hat{\mathrm{B}}=\hat{\Phi} \cdot \nabla \hat{\Phi}=-\hat{\mathrm{R}} / \mathrm{R}$. The "curvature" drift velocity in three dimensions is:
$\mathrm{v}_{\mathrm{c}}=\frac{1}{\mathrm{qB}^{2}}\left(\mathrm{mv}_{\| \mathrm{gc}}^{2} \frac{\hat{\mathrm{R}}}{\mathrm{R}}\right) \times \mathrm{B}$

Which is directed either into or out of the page depending the sign of the electric charge q. The centripetal force reads:

$\mathrm{F}_{\mathrm{cp}}=\mathrm{mv}_{\| \mathrm{gc}}^{2} \frac{\hat{\mathrm{R}}}{\mathrm{R}}$

\subsection{Time-Varying Electric Field Strength $\mathbf{E}$}

For slightly time-varying electric field E, the electric and magnetic field strength would read:

$$
\begin{aligned}
& \mathrm{E}(\mathrm{x}, \mathrm{t})=\mathrm{E}_{0}(\mathrm{x}) \exp (\mathrm{i} \Omega \mathrm{t}) \\
& \mathrm{B}(\mathrm{z})=\mathrm{Bz}
\end{aligned}
$$

We notice that Equation 16 would result in an equation of motion as follows:

$$
\begin{aligned}
& \frac{\mathrm{d}^{2} \mathrm{v}_{\perp}}{\mathrm{dt}^{2}}=-\Omega_{\mathrm{c}}^{2}\left[\mathrm{v}_{\perp}-\frac{\mathrm{q}}{|\mathrm{q}|} \frac{\mathrm{i} \Omega}{\Omega_{\mathrm{c}}} \frac{\mathrm{E}_{\perp}(\mathrm{x}, \mathrm{t})}{\mathrm{B}(\mathrm{z})}\right] \\
& \equiv-\Omega_{\mathrm{c}}^{2}\left[\mathrm{v}_{\perp}-\tilde{\mathrm{v}}_{\|}\right]
\end{aligned}
$$

Which obviously describes a harmonic motion. With the assumption that $\mathrm{E}$ is slight varying in time, the "polarization" drift velocity takes the form:

$\mathrm{v}_{\mathrm{p}}=\frac{\mathrm{m}}{\mathrm{qB}^{2}} \frac{\mathrm{dE}_{\perp}(\mathrm{x}, \mathrm{t})}{\mathrm{dt}}=-\frac{1}{\Omega_{\mathrm{c}} \mathrm{B}}\left(\frac{\mathrm{d}}{\mathrm{dt}} \mathrm{V}\right) \times \mathrm{B}$

where, $\mathrm{V}$ is the generic drift velocity and the direction is defined by the sign of the electric charge $q$, as given in Equation 7. This drift motion obviously differs from other drifts. It even can inhere in it other drift motions. It is not allowed to continue indefinitely. Examples for temporarily varying electric field $\mathrm{E}$ are not rare. For example an oscillatory $E$, which would result in $v_{p}$. The latter oscillates normally out of phase. Because of the mdependence, this drift is also called "inertia" and can be neglected for light particles. Then, as given above, the "polarization" drift force reads:

$\mathrm{F}_{\mathrm{p}}=-\Omega^{2} \mathrm{~m}\left[\mathrm{r}_{\perp}-\tilde{\mathrm{r}}_{\|}\right]$

\subsection{Time-Varying Magnetic Field Strength B}

According to Faraday's law, the time-varying magnetic field $\mathrm{B}$ generates an electric field $\mathrm{E}, \mathrm{dB} / \mathrm{dt}=$ 
$-\nabla \times$ E. Considering a vector 1 along the perpendicular trajectory, then the time evolution of the particle's kinetic energy reads:

$$
\frac{1}{2} \mathrm{~m} \frac{\mathrm{d}}{\mathrm{dt}} \mathrm{v}_{\perp}^{2}=\mathrm{qE} \cdot \frac{\mathrm{dI}}{\mathrm{dt}}
$$

Which leads to change the initial kinetic energy to:

$$
\frac{1}{2} \mathrm{~m}\left(\mathrm{v}_{\perp, \mathrm{f}}^{2}-\mathrm{v}_{\perp, \mathrm{i}}^{2}\right)=2 \pi \frac{\mu}{\Omega_{\mathrm{c}}} \frac{\mathrm{dB}}{\mathrm{dt}}
$$

The r. h. s. gives the energy added to the initial kinetic energy by time-varying magnetic field $B$. The "grad E" drift force reads:

$$
\delta \mathrm{F}_{\mathrm{VE}}=2 \pi \frac{\mu}{\Omega_{\mathrm{c}} \mathrm{r}} \frac{\mathrm{dB}}{\mathrm{dt}}
$$

\subsection{Generic Drifts}

One can think of two other cases, in which both fields are either spatially or temporarily varying. That one is spatially or temporarily varying while the other is the opposite would give two additional cases. Various drift motions are possible under action of the generic force $F=F_{\|}$ $\cos (\theta)+F_{\perp} \sin (\theta)$. The component $F_{\|} \cos (\theta)$ seems to add to the acceleration along $\mathrm{B}$ and the component $\mathrm{F}_{\perp} \sin (\theta)$ add to the drift motion velocity. This leads to the conclusion that the final acceleration under the influence of the field interactions is composed of three parts:

- Direct acceleration under the influence of $F_{\|} \cos (\theta)$

- Drift acceleration by $F_{\perp} \sin (\theta)$ and

- Gyration by B itself

In Equation 18, E can be replaced by the summation of the forces given in Equation 21-31. The various hypothetical astrophysical sources would sanctify to conclude that the cosmic particles accumulatively gain their final energy through the participation in a wide range of various interactions.

\section{MATERIALS AND METHODS}

In the present work, we utilized models in order to find out formalism estimating the potential acceleration of the cosmic charged, massive particles when passing through diiferent typles of plasma fields. The mathematical methods are basically based on applying fundamental rules given by electromagnetic interactions with interstellar objects. Enrico Fermi apparwently drawn the same line, couple decades ago. The materials available to this work are rich litrature, previous investigations and successful models. On top of this list, we put the observatiobal confirmation of the relation governing the cosmic ray energies.

\section{RESULTS}

In the present work, we poposed a theoretical model based on the interaction of the cosmic charged particle with the ambient electromagnetic field. The electric field is only able to accelerate the cosmic charged particles to extreme energies up to $10^{20} \mathrm{eV}$. The configuration of the electromagnetic field has an essential role in the acceleration mechanism, as the maximum acceleration can be obtained when the electric field $\mathrm{E}$ and the magnetic field B are parallel to each other. In addition to, the direction of the motion of the charged particle itself is strongly influenced by the electric and magnetic field state. The plausible astrophysical sources of UHECRs introduce miscellaneous cases of the electromagnetic field. Therefore, some sorts of cosmic drift motions could be pivotal sources for acceleration. Moreover, the spatially varying electromagnetic field could be considered, where the electromagnetic fields of the astrophysical environment is locally conserved. In this regard Equation 9 shows the twice of energy introduced by the Hillas (1984) argument. The extension of the present model to include the Fermi acceleration mechanisms is applicable. This will verify the characteristic power law of the UHECs accelerated by the electric field or the direct acceleration (Tawfik et al., 2010). According to the present work, we could assert that there are some physical conditions could be taken into account besides the direct and Fermi acceleration mechanisms. Configuration of the cosmic electromagnetic fields, different drift motions and the combination of the direct and Fermi acceleration mechanisms.

\section{DISCUSSION}

The Fermi mechanism (Fermi and Yang, 1949) is to be illustrated as the cosmic charged particles are accelerated through random elastic collisions with the irregularities of the interstellar objects, such as magnetic clouds and matter clumps. Therefore, the sources for the ultra high energy are likely time-dependent magnetic and induced electric fields. The energy that the cosmic charged particles would gain depends only on $u$, the velocity of the magnetic clouds, but not on the magnetic field strength $B$ and/or electric charge q. Because of the 
rare elastic collisions, this mechanism is criticized to be too slow to attain UHECR. The other concern is going on the effects of Coulomb interactions on the energy loss of the cosmic charged particles injected at relatively low energy. The Coulomb interactions use a large portion of this gained energy. Astrophysical objects with turbulent magnetic fields would offer a realization of matter clumps, that homogeneously distributed in the galactic space and move with the Alfven wave velocity vA. The shock scatterings through the expansion of matter matter clumps flowing at speeds larger than the speed of sound, like remnant supernovae, are likely frequent. The acceleration according to this mechanism is much more efficient than the stochastic one.

So far, we summarize that the electromagnetic drift motions would be possible sources to accelerate cosmic charged particles. Since various types of field interactions can also exist in cold plasmas, nonrelativistic formalism for different the types of electromagnetic interactions is given. The translation to a relativistic version is straightforward. The elastic and shock scatterings, the Fermi acceleration mechanisms, play their essential role in this model. Furthermore, general configurations for both electromagnetic fields are taken into consideration. It has been conjectured that various interaction types might be possible in one astrophysical object. Examples on drift forces depending on the nonuniformality and nonhomogeneity of both electromagnetic fields have been discussed. The final energy, that the cosmic charged particle would gain, is assumed to stem from different sources. Fermi mechanisms are obvious sources. Plasma field interactions are suggested as additional sources. Obviously, the latter date back to eighties of last century, when Hillas introduced his wellknown graph (Hillas, 1984) and a very special configuration that magnetic and electric forces are perpendicular to each other has been studied. This apparently would limit the gyration to a concrete minimum value.

The present model is applied on different astrophysical objects, in order to calculate the energy gained by a singly charged and massive particle (Tawfik et al., 2010). It has been found that the order of the electromagnetic field acceleration is zero-order, while Fermi mechanisms apparently describe first and second-order relative velocities (Tawfik and Saleh, 2010).

\section{CONCLUSION}

The Ultra High Energy Cosmic Rays (UHECRs) are still a vibrant subject of intense debate, where their origin and acceleration mechanisms are not known with certain. Our concern in this work is to study the acceleration of UHECRs up to $10^{20} \mathrm{eV}$ and beyond starting from low energies, i.e. bottom-up scenario. The plausible sources of UHECRs exhibit different physical conditions of electromagnetic fields, which we exploited. Obviously, the equation of motion of the cosmic charged particle is in terms of the cosmic electric field only. Consequently, the electric field has an essential role in the acceleration process of UHECRs. The unipolar inductor sources like pulsars, magnetars, planet magnetospheres and the electric fields in quasiperpendicular shock waves can provide direct candidates for electric fields. The configuration of the electromagnetic field can strongly affect the cosmic charged motion and hence, the maximum acceleration is achievable when the electric field $\mathrm{E}$ is perpendicular to the magnetic one $\mathrm{B}$. The various drift motions of the cosmic particles due to the different astrophysical plasma aspect could play intrinsic role in the acquired energy. Therefore, in the forthcoming work (Tawfik et al., 2010), we calculate the attainable energy in each case. The extension of the generic model to incorporate the second order Fermi acceleration process could raise the cosmic particle energy.

\section{REFERENCES}

Abraham, J., P. Abreu, M. Aglietta, C. Aguirre and D Allard, 2007. Correlation of the highest-energy cosmic rays with nearby extragalactic objects. Science, 318: 938-943.

Blandford, R.D. and D. Eichler, 1987. Particle acceleration at astrophysical shocks: A theory of cosmic ray origin. Astrophys. J., 154: 1-75. DOI: 10.1016/0370-1573(87)90134-7

Boris, V.S., 2013. Plasma Astrophysics, Part II: Reconnection and Flares. 2nd Edn., Springer, New York, ISBN-10: 1461442958, pp: 413.

Fermi, E. and C.N. Yang, 1949. Are mesons elementary particles? Phys. Rev., 76: 1739-1743. DOI: 10.1103/PhysRev.76.1739

Ghisellini, G., G. Ghirlanda, F. Tavecchio, F. Fraternali and G. Pareschi, 2008. Ultra-high energy cosmic rays, spiral galaxies and magnetars. MNRAS, 390: L88-L92. DOI: 10.1111/j.1745-3933.2008.00547.x

Greisen, K., 1966. End to the cosmic-ray spectrum? Phys. Rev. Lett., 16: 748-750. DOI: 10.1103/PhysRevLett.16.748

Hillas, A.M., 1984. The origin of ultra-high-energy cosmic rays. Ann. Rev. Astron. Astrophys., 22: 425444. DOI: 10.1146/annurev.aa.22.090184.002233 
Inoue, S., 2008. Origin of ultra-high energy cosmic rays in the era of auger and telescope array. J. Phys. Conf. Ser., 120: 062001-062001. DOI: 10.1088/1742-6596/120/6/062001

Kelley, M.C., 2009. The Earth's Ionosphere: Plasma Physics and Electrodynamics. 2nd Edn., Academic Press, Boston, ISBN-10: 9780120884254, pp: 576.

Klein, K.L., 2003. Energy Conversion and Particle Acceleration in the Solar Corona. 1st Edn., SringerVerlag, Berlin, New York, ISBN-10: 3540002758, pp: 319.

Kotera, K., A.V. Olinto, 2011, The astrophysics of ultrahigh-energy cosmic rays. Ann. Rev. Astronomy Astrophysics, 49: 119-153. DOI: 10.1146/annurevastro-081710-102620

Marconi, A., 2006. Astron. Astrophys, 448: 921-921.

Murase, K. and J.F. Beacom, 2010. Very-high-energy gamma-ray signal from nuclear photodisintegration as a probe of extragalactic sources of ultrahighenergy nuclei. Cornell University Library.

Nagano, M. and A.A. Watson, 2000. Observations and implications of the ultrahigh-energy cosmic rays. Rev. Modern Phys., 72: 689-732. DOI: 10.1103/RevModPhys. 72.689

Parkes, G.K., 2004. Physics of Space Plasmas: An Introduction. 2nd Edn., Westview Press, Boulder, Colo., ISBN-10: 0813341299, pp: 597.

Paul, M.B., 2006. Fundamentals of Plasma Physics. 1st Edn., Cambridge University Press, Cambridge, ISBN-10: 0521821169, pp: 628.
Prescod-Weinstein, C., N. Afshordi and M.L. Balogh, 2009. Stellar black holes and the origin of cosmic acceleration. Phys. Rev. D., 80: 043513-043513. DOI: 10.1103/PhysRevD.80.043513

Schlickeiser, R. and F. Jenko, 2010. Cosmic ray transport in non-uniform magnetic fields: Consequences of gradient and curvature drifts. J. Plasma Phys. Bf, 76: 317-327. DOI: 10.1017/S0022377809990444

Takeda, M., N. Hayashida, K. Honda, N. Inoue and K. Kadota et al., 1998. Extension of the cosmic-ray energy spectrum beyond the predicted greisenZatsepin-Kuz'min cutoff. Phys. Rev. Lett., 81: 1163-1166. DOI: 10.1103/PhysRevLett.81.1163

Tawfik, A. and A. Saleh, 2010. Orders of Fermi- and Plasma-Accelerations of Cosmic Rays. Cornell University Library.

Tawfik, A., A. Saleh, M.T. Ghoneim and A. Hady, 2010. Acceleration and particle field interactions of cosmic rays II: Calculations. Cornell University Library.

Teshima, M., 2001. AGASA results and telescope array project. Prog. Theor. Phys. Suppl., 566: 184-194. DOI: $10.1063 / 1.1378631$

Yoshida, S. and H. Dai, 1998. The extremely high energy cosmic rays. J. Phys. G: Nucl. Particle Phys., 24: 905-905. DOI: 10.1088/0954-3899/24/5/002

Zatsepin, G. and V.A. Kuzmin, 1966. Upper limit of the spectrum of cosmic rays. J. Exp. Theoret. Phys. Lett., 4: 78-78. 